\title{
La demande de bois-énergie à Ouagadougou : esquisse d'évaluation de l'impact physique et des échecs des politiques de prix
}

\section{Boukary Ouedraogo}

\section{(2) OpenEdition}

Journals

Édition électronique

URL : http://journals.openedition.org/developpementdurable/4151

DOI : 10.4000/developpementdurable.4151

ISSN : 1772-9971

Éditeur

Association DD\&T

Référence électronique

Boukary Ouedraogo, « La demande de bois-énergie à Ouagadougou : esquisse d'évaluation de l'impact physique et des échecs des politiques de prix », Développement durable et territoires [En ligne], Varia (2004-2010), mis en ligne le 20 mars 2006, consulté le 19 avril 2019. URL : http://

journals.openedition.org/developpementdurable/4151; DOI : 10.4000/developpementdurable.4151

Ce document a été généré automatiquement le 19 avril 2019

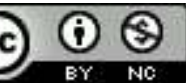

Développement Durable et Territoires est mis à disposition selon les termes de la licence Creative Commons Attribution - Pas d'Utilisation Commerciale 4.0 International. 


\title{
La demande de bois-énergie à Ouagadougou : esquisse d'évaluation de l'impact physique et des échecs des politiques de prix
}

\author{
Boukary Ouedraogo
}

1 Le Burkina Faso comptait entre 1980 et 1985, 14,107 millions d'hectares de forêts et de zones boisées soit $52 \%$ de sa superficie totale (Sharma, 1992). Le rapport de la Banque Mondiale (1992) sur le développement estime à 80000 hectares le niveau de déboisement annuel soit de 1,7\% du total de ses forêts; ce taux étant près de trois fois celui de l'Afrique qui est de 0,5\%. En effet, la rareté des revenus monétaires en milieu rural, et l'avancée du désert depuis le Sahel, ont engendré de forts courants migratoires des populations. Ces migrations sont orientées des campagnes vers les villes et des zones sèches et dégradées vers celles plus humides du pays, amplifiant la pression sur les ressources forestières et entrainant une forte dégradation du couvert végétal et la désertification. Les ressources ligneuses s'apparentent à bien des égards à des ressources à accès libre qui satisfont à des besoins primaires: bois-énergie pour la cuisson des repas quotidiens et la production artisanale, bois de service pour construire des maisons et des hangars, bois d'?uvre pour fabriquer des meubles (lits, chaises, autres produits artisanaux), le bois d'?uvre d'art (sculptures diverses), la pharmacopée et l'alimentation du bétail. Au cours des vingt dernières années, les superficies cultivées ont augmenté en moyenne chaque année de 2,69\% (MARA/DSAP, 1970-1995) presque à un rythme identique à celui de la population (2,8\% en 1998).

2 L'étude de la demande du bois-énergie à Ouagadougou présente une certaine hétérogénéité au niveau des modes d'exploitation des ressources ligneuses: la filière organisée et la filière non organisée, qui présente chacune des caractéristiques assez particulières :

3 (a) La filière organisée est celle qui est basée sur le bois exploité dans les chantiers aménagés. Selon la FAO (1995), l'expression « Aménagement des forêts » recouvre tous les 
aspects administratifs, économiques, juridiques, sociaux, techniques et scientifiques de la conservation et de l'utilisation des forêts. Il s'agit donc de l'application de la foresterie à une aire géographique donnée en vue d'en assurer aux communautés les avantages qui en dérivent, et aux gestionnaires un revenu optimal et soutenu. La zone d'aménagement correspond à l'endroit où est pratiqué l'aménagement. Selon le dernier rapport général de la gestion durable des forêts au Burkina (FAO, 1996), "L'aménagement forestier pour l'approvisionnement de Ouagadougou en bois de feu et la sauvegarde de l'environnement a été mis en ?uvre en 1986 à travers le «Projet Aménagement des Forêts Naturelles" par l'Etat Burkinabé avec l'appui financier du PNUD et l'assistance technique de la FAO. Aujourd'hui près de 667600 hectares sont aménagées dont les 50\% sont autonomes et gérées par les unions et groupement de gestion forestières. 202400 hectares sont en instance d'aménagement. » Ces zones aménagées comptent aujourd'hui près de 8 Unions de Gestion Forestière officiellement reconnues qui regroupent plus de 250 Groupements de Gestion Forestière. L'exploitation du bois dans les zones aménagées se fait suivant les normes sylvicoles. Les bûcherons sont formés en techniques sylvicoles, notamment dans les techniques de coupe, et sont encadrés par un ingénieur forestier appelé Directeur technique, qui assure la gestion technique de l'exploitation durable de la forêt. Les exploitants de cette filière opèrent dans un rayon minimal de 70 kilomètres de Ouagadougou. La spécificité de ces zones de production est qu'elles prélèvent des écotaxes (la taxe forestière et la redevance forestière): les prix au producteur sont administrés en amont de la filière. La rémunération des bûcherons est fixée dans les zones aménagées de production du bois-énergie à 1100 FCFA le stère de bois coupé. A ce prix il faut ajoutée la taxe forestière d'une valeur de $300 \mathrm{FCFA} / \mathrm{stère,} \mathrm{la}$ redevance forestière d'une valeur de $600 \mathrm{FCFA} /$ stères et du fonds de roulement villageois d'une valeur de $200 \mathrm{FCFA} /$ stère. Le prix au producteur revient à $2200 \mathrm{FCFA} /$ stère. Les grossistes transporteurs du bois de feu (gros camionneurs en général) fixent librement le prix aux détaillants selon la saison (sèche ou pluvieuse) qui détermine les difficultés de la pratique de cette activité. On observe une forme de marché hybride dans cette filière organisée où en amont l'administration des prix au producteur se greffe avec une fixation libre des prix au détaillant et au consommateur.

4 (b) La filière non organisée est celle qui exploite le bois dans les zones non aménagées. C'est une filière où les exploitants (en grande partie constitués de charretiers et de cyclistes) coupent le bois dans leur village d'origine et environnant, et le transportent à Ouagadougou où il est vendu à des ménages, à des artisans ou à des détaillants. Les exploitants de cette filière opèrent dans un rayon maximum de 70 kilomètres de la Ouagadougou. La spécificité dans cette d'exploitation est que les exploitants échappent aux écotaxes payés en amont de la filière organisée. Les bûcherons acheminent euxmêmes le bois-énergie jusqu'à Ouagadougou pour la vente. Cette filière est très importante au regard du nombre de charretiers et de cyclistes de plus en plus croissant, mus par un environnement précaire et fragile du point de vue économique. Les exploitants de cette mode de production achètent simplement des permis de coupe et de transport du bois auprès des services forestiers, coupent, transportent et vendent le bois de feu en ville après de longs marchandages. Cette forme de marché est plus proche du marché de concurrence parfaite car le nombre de plus en plus croissant des exploitants de cette filière donnent plus de choix de aux consommateurs (Ouédraogo, 2002). Le choix de Ouagadougou comme zone d'étude s'explique par plusieurs raisons dont :

6 Primo, la région de Ouagadougou a été déterminée par les géographes de l'Université d'Amsterdam comme faisant partie des zones urbaines semi-arides de l'Afrique de l'Ouest 
où les aléas et la variabilité climatiques sont les plus marqués et où les densités et les mouvements démographiques sont les plus forts (Dietz, Veldhuizen, 1998). La région de Ouagadougou connaît depuis les années 80 une forte urbanisation et, au cours des 25 dernières années, la population de la ville a connu une augmentation de près de $300 \%$ avec une forte croissance démographique de l'ordre de $6,2 \%$ par an et a reçu annuellement en moyenne 80000 migrants accroissant très rapidement les besoins en énergie domestiques (INSD, 1970-1996).

7 Secondo, une importante filière de bois-énergie s'est développée pour approvisionner Ouagadougou en bois-énergie. La concentration de la population et l'importance des activités artisanales utilisatrices du bois-énergie (production de bière traditionnelle à base de sorgho rouge, fonderie, restauration etc.) se répercutent sur cette filière qui ne cesse de grossir chaque année : près de 50000 bûcherons, quelques projets forestiers dont le projet «BKF, aménagement des forêts naturelles" et le projet "Gestion forestière intégrée ", environ 10000 artisans utilisateurs de l'énergie ligneuse comme consommation intermédiaire et approximativement et 700000 consommateurs potentiels ménagers en 2000 (Ouédraogo, 2002).

8 Tertio, selon la carte de la situation agro-écologique (PANE, 1994), la région du Centre avec Ouagadougou comme chef lieu, est fortement déficitaire en ressources ligneuses enregistrant à elle seule un déficit de $900000 \mathrm{~m}^{3}$ de bois-énergie, soit un déficit deux fois plus élevé que celui constaté à l'échelle nationale et soumise à de fortes érosions hydriques et éoliennes (PANE,1994). En effet, les superficies boisées de la Région du Centre dont Ouagadougou est le chef lieu sont passées de 38408 kilomètres carrés en 1980 à 13255 kilomètres carrés en 1995, soit une réduction de 25153 kilomètres carrés, soit l'équivalent de 66\% en 15 ans (Revue des Dépenses Publiques/M.E.F., 1996). Une décennie auparavant, paraissait dans les éditions Unasylva un ouvrage intitulé « Une ville africaine en crise d'énergie» (Chavin, 1981) avec pour référence la ville de Ouagadougou, et ceci pour souligner la crise des énergies de cuisson que vivent les consommateurs de celle-ci.

Cet article vise à : 1) évaluer les potentialités forestières de l'aire d'approvisionnement de Ouagadougou en bois-énergie; 2) estimer les coefficients de consommation et les quantités consommées pour les différents segments de consommateurs du bois-énergie; 3) évaluer en l'an 2000, l'impact de la demande globale en bois-énergie de cette ville sur le patrimoine forestier de l'aire d'approvisionnement; 4) faire une esquisse d'évaluation des échecs des politiques de prix $d u$ bois-énergie. Pour bien conduire cette étude, nous allons successivement présenter la démarche méthodologique adoptée et les sources statistiques utilisées, l'aire d'approvisionnement et évaluer son potentiel ligneux durablement exploitable, les évaluations des coefficients de consommations et la consommation globale de cette ville, évaluer l'impact de cette consommation sur les ressources forestières, et enfin analyse les causes des échecs des politiques de prix du bois-énergie à Ouagadougou.

1. Sources statistiques utilisées

10 La conduite de cette étude, nécessite la connaissance des quantités consommées. Il faut disposer de statistiques sur les différents segments de consommateurs du bois-énergie. C'est pourquoi, nous présentons les sources statistiques utilisées et les méthodes d'évaluation des quantités consommées. Quatre sources statistiques ont été utilisées pour réaliser cette étude.

Enquête sur les dépenses des ménages (INSD/UEMOA, 1996) 
11 La source statistique utilisée concerne les données sur les dépenses des ménages de Ouagadougou, la capitale du Burkina Faso, collectées dans le cadre du projet d'harmonisation des statistiques sur les prix à la consommation dans les sept pays de l'Union Économique et Monétaire Ouest Africain (UEMOA), réalisées par les Instituts Nationaux de la Statistique de ces pays en 1996. L'objectif principal de ces enquêtes harmonisées est de surveiller de manière multilatérale les performances économiques par la détermination des pondérations pour le calcul de l'Indice Harmonisé des Prix à la Consommation (IHPC) dans les pays de cette zone économique et monétaire. La base de données a été réalisée grâce à une équipe d'experts de l'Institut National de la Statistique et des Études Économiques de la France, des experts d'EuroCost, en collaboration avec les experts nationaux des Instituts Nationaux de la Statistique des pays de l'UEMOA. La taille de l'échantillon a été fixée à 1008 ménages de cette ville (INSD, 1998). La méthode de sondage mise en ?uvre est celle d'un sondage stratifié à deux degrés. L'enquête s'est déroulée du 12 mars au 6 juin 1996. Les variables collectées concernent essentiellement les caractéristiques des ménages, celles de leurs habitations et les différents postes de dépenses effectués par ceux-ci quotidiennement.

Enquêtes sur la demande des artisans de Ouagadougou (mai, 2000)

Les enquêtes sur la consommation des artisans ont ciblé les cinq principales catégories d'artisans, qui utilisent le bois-énergie comme facteur de production. Nous avons réalisé ces enquêtes en mai 2002 en collaboration avec le Programme Régional pour le sous Secteur des Énergies Traditionnelles (RPTES) Les populations statistiques Concernées sont par catégorie : les Fondeurs de bronze qui regroupent les artisans fabriquant des objets d'art en bronze; les Fondeurs d'aluminium, qui regroupent les artisans fabriquant des marmites et d'autres types d'ustensiles de cuisine en aluminium; les " Dolotières » sont des femmes qui fabriquent la bière traditionnelle de sorgho rouge appelée « dolo »; les Restaurateurs qui sont les gargotes généralement tenues par des femmes vendant leur repas sous des hangars; les Grilleurs de viandes qui sont vendeurs de viande grillée, le plus souvent avec des grands foyers traditionnels et des fourneaux à charbon de bois. Ces différentes catégories d'artisans ont des activités homogènes ${ }^{1}$ et la seule différence est souvent leur taille, leur capacité de pénétration du marché. Nous avons pu toucher lors de ces enquêtes, 375 artisans. Le tableau 1 donne les tailles des échantillons des catégories d'artisans.

Tableau 1: Tailles des échantillons.

\section{Population cible Effectif en 2000 Taille effective}

\begin{tabular}{lcc}
\hline Fondeurs de bronze & 231 & 50 \\
Fondeurs de marmites & 240 & 50 \\
Dolotières & 1013 & 154 \\
Grilleurs de viandes & 2715 & 50 \\
Restaurateurs & 2096 & 71 \\
Total & 6295 & 375 \\
\hline \hline
\end{tabular}

Sources : Évaluation des tailles des échantillons à partir de la formule de Gauss. 
13 L'homogénéité de l'activité au sein de chaque catégorie d'artisans nous permet de retenir une taille de l'échantillon en faisant un tirage raisonné dans chaque catégorie d'artisans de sorte à faire varier la taille des exploitants afin de couvrir les différentes tailles d'exploitations qui existent dans l'environnement de Ouagadougou. Cette manière de procéder garantit une certaine représentativité de l'échantillon au regard de l'homogénéité du point de vue des techniques et méthodes de production de l'activité artisanale.

Les enquêtes de pesées auprès des intermédiaires commerciaux (2002)

L'objectif de ces enquêtes a été de saisir le prix au kilogramme du bois-énergie dans les différents secteurs et quartiers de la ville de Ouagadougou : ce qui permet de convertir les dépenses en volume de bois-énergie. Ces enquêtes se sont déroulées à deux niveaux : au niveau des détaillants du bois-énergie, elles ont consisté à peser les différents tas de bois de feu ou de charbon, vendus par les détaillants dans les secteurs de la ville de Ouagadougou; au niveau des grossistes-transporteurs (charretiers et camionneurs), l'enquête a consisté à saisir les prix minimum et maximum auxquels ceux-ci écoulent leurs chargements, et les capacités de charge des véhicules utilisés pour le transport du bois-énergie.

Les tailles des échantillons par type de vendeurs de bois-énergie sont données dans le tableau suivant :

Tableau 2:Tailles des échantillons.

\begin{tabular}{|c|c|c|c|c|}
\hline $\begin{array}{c}\text { Types de } \\
\text { vendeurs }\end{array}$ & $\begin{array}{c}\text { Détaillants } \\
\text { bois de } \\
\text { feu }\end{array}$ & $\begin{array}{c}\text { Détaillants } \\
\text { de charbon } \\
\text { de bois }\end{array}$ & Charretier & Camionneur \\
\hline $\begin{array}{c}\text { Taille de } \\
\text { l'échantillon }\end{array}$ & 18 & 31 & 10 & 15 \\
\hline
\end{tabular}

Source : Résultats des enquêtes de pesée du bois-énergie à Ouagadougou (mai, 2000)

Inventaire forestier dans la région de Ouagadougou

A l'initiative du projet BKF, le laboratoire de la FAO appuyé par l'Institut Géographique du Burkina (IGB) et financé par le PNUD, ont entrepris en 1990, une délimitation géographique et administrative, ainsi qu'une caractérisation du potentiel forestier de l'aire d'approvisionnement de la ville de Ouagadougou en bois-énergie. Les données de cette investigation ont permis d'avoir des données chiffrées sur l'occupation des sols dans cette région et la densité des ressources forestières. Ces données ont été très déte rminantes dans l'étude d'impact.

2. Méthodologie

17 Les méthodes d'évaluation des indicateurs de consommations des usagers du bois-énergie seront présentées, ainsi que la méthode de calcul de la demande globale de ceux-ci.

2.1. Méthodes d'évaluation des coefficients de consommation

Deux méthodes d'évaluation des quantités consommées de bois-énergie existent. La méthode la plus utilisée est celle de la pesée; elle a été utilisée jusqu'en 1995 par plusieurs auteurs (qui ont travaillé) dans le secteur des énergies de cuisson (Ouédraog, 1974 ; De Backer, 1980 ; Chavin, 1981 ; ESMAP, 1987 ; Mostert, 1989 ; Zida, 1991 ; IBE, 1992 ). La deuxième méthode, qualifiée de "méthode de la conversion des dépenses", a été élaborée et utilisée pour la première fois par Ouédraogo (1996). 
La méthode de la pesée construire un échantillon représentatif des ménages et/ou des artisans et à peser les quantités de bois-énergie disponibles pour le ménage (ou l'artisan) avant et après chaque cuisson (ou chauffage) pendant une certaine période. Les résultats de ces pesées permettent selon la taille des ménages (artisans) touchés, de calculer le coefficient (ou l'indicateur) de consommation par tête d'habitant (ou par artisan). Cet indicateur est donné en kilogramme par jour et par habitant (et/ou par artisan). La pertinence de la méthodologie d'établissement de cet indicateur et les difficultés d'évaluation en amont des quantités produites et commercialisées du bois-énergie confèrent à ce coefficient une grande utilité dans les études prospectives du secteur bois-énergie. Malgré la pertinence de la méthode de la pesée, elle a des limites qui sont entre autres que pour être représentatif, le coefficient issu de la pesée doit être une moyenne de pesées de la saison sèche et des pesées de la saison pluvieuse; et cela demanderait beaucoup de temps pour constituer un coefficient de consommation représentatif par la méthode de la pesée. Cette méthode est très coûteuse car elle nécessite non seulement un suivi régulier des ménages (ou artisans) ciblés pour ces pesées, mais aussi des pesées pendant un mois au moins (un mois) sur l'ensemble de l'échantillon. C'est pourquoi, nous privilégions l'utilisation de méthodes plus efficaces, flexibles et moins onéreuses.

La méthode de « la conversion des dépenses »

La méthode de la «conversion des dépenses " de consommation de bois-énergie en volume de bois-énergie consiste à diviser la dépense par tête d'habitant pour une période donnée par le prix moyen du kilogramme de bois-énergie de la même période. La procédure de calcul est la suivante. On divise d'abord la somme des dépenses journalières par ménage pour l'achat du bois de feu par le nombre de personnes de chaque ménage, pour obtenir la dépense par jour et par habitant en bois de chauffe. Ensuite on rapporte ce résultat au prix moyen du kilogramme du bois de chauffe sur le marché pour chiffrer l'indicateur de consommation domestique du bois de feu (en kg/jour/habitant).

21 Cette méthode nous a semblé beaucoup plus efficace et moins coûteuse pour les raisons suivantes. Tout d'abord, elle utilise les prix moyens du kilogramme de bois-énergie, prix issus des pesées mensuelles du bois-énergie auprès des intermédiaires commerciaux de ce combustible, l'Institut National de la Statistique et de la Démographie effectuant des pesées mensuelles du bois-énergie dans les différents quartiers et secteurs de la ville de Ouagadougou pour établir les prix moyens consommateur du kilogramme du combustible ligneux. Ensuite, les prix moyens mensuels du bois-énergie contiennent toutes les informations liées à la rareté saisonnière du combustible et donc garantissent la pertinence et la représentativité du coefficient issu de cette méthode par rapport aux variations saisonnières des prix. Enfin, la connaissance des dépenses de consommation de ce combustible suffit pour évaluer efficacement les quantités consommées et donc d'établir le coefficient de consommation. Et puisque nous disposons dans notre base de données des dépenses annuelles des ménages en énergies de cuisson, la méthode de la « conversion des dépenses » permet grâce au prix moyen du kilogramme des différentes énergies de cuisson, de passer aux volumes consommés de celles-ci.

2.2. Méthodes d'évaluation de la consommation.

22

Pour les ménages, la seule connaissance de la dépense totale des ménages pour une période donnée suffit grâce à la méthode de la conversion des dépenses pour évaluer la consommation globale pour la période considérée. Cependant, pour les artisans, en plus 
de la connaissance des coefficients de consommation par artisan et par jour, il faut connaître la fréquence hebdomadaire de l'activité pour pouvoir évaluer leur consommation globale. La demande globale ou consommation globale de la ville de Ouagadougou sera la somme des consommations des ménages et celle des artisans pour une période considérée.

3. Aires d'approvisionnement de Ouagadougou et potentialités forestières3.1.

Délimitation géographique et administrative de la région

L'identification, la délimitation et la caractérisation de la zone d'approvisionnement de Ouagadougou en bois-énergie a été l'initiative du Projet FAO/BKF/89/011 « Aménagement des Forêts Naturelles pour la Sauvegarde de l'Environnement et la Production du Boisénergie » financé par le Programme des Nations Unies pour le Développement (PNUD) et exécuté par l'Organisation des Nations Unies pour l'Alimentation et l'Agriculture (FAO). L'étendue maximale susceptible d'approvisionner la capitale en bois de feu, en fonction des réserves forestières et des distances de transport, représente une superficie de six millions d'hectares et se délimite ainsi qu'il suit: au Sud, par la frontière du Ghana, approximativement le long du parallèle $11^{\circ} \mathrm{Nord}$; au Nord par le parallèle $13^{\circ}$ Nord, au delà duquel les formations boisées deviennent très rares ; à l'Est, par le méridien $0^{\circ}$, situé à environ $160 \mathrm{Km}$ de Ouagadougou ; à l'Ouest, par le fleuve Mouhoun, qui coule à peu près le long du méridien $3^{\circ}$ Ouest.

Cette zone est habitée par une importante population à prédominance rurale qui pratique des activités agropastorales de manière si extensive qu'elles exercent une forte pression sur les formations naturelles. Elle a été délimitée dans un rayon de 150 kilomètres de Ouagadougou pour tenir compte de la distance maximale que les grossistes-transporteurs du bois acceptent de parcourir pour rentabiliser leur activité. Cette délimitation a en outre pris en compte les aspects de la demande de bois-énergie, les capacités de régénération des massifs forestiers considérés et des possibilités de reconstitution, de conservation et protection de ceux-ci.

La carte géographique ci-dessous présente les limites de la zone d'approvisionnement de Ouagadougou en bois-énergie, les chefs lieu des provinces, spécifie les zones d'intervention des types de transports, et la disponibilité des ressources ligneuses dans la région. 


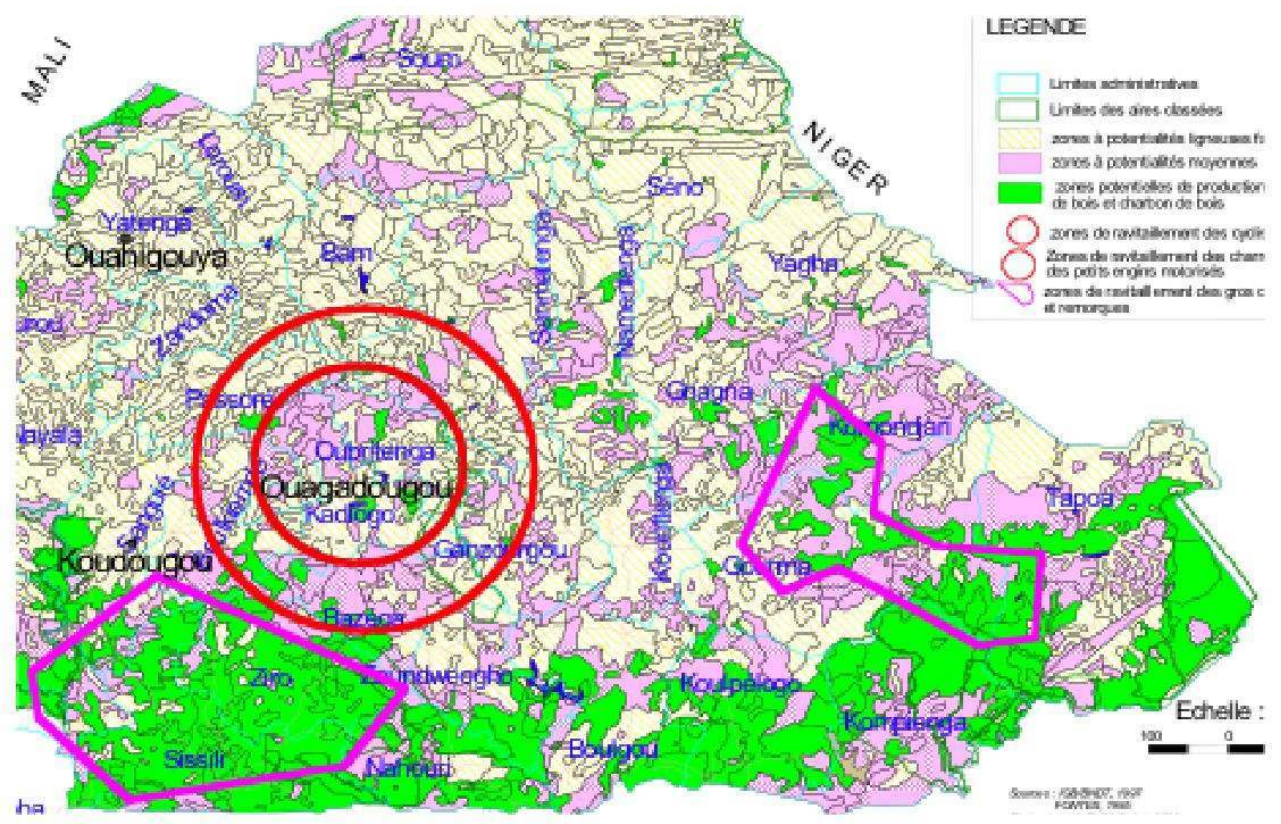

La délimitation géographique de cette zone permet d'identifier les entités administratives qui s'y trouvent. On distingue les provinces suivantes avec les superficies respectives dans la zone et les chefs lieu de provinces dans le tableau 3.

Tableau 3 : Définition administrative de la zone

\begin{tabular}{lcc}
\hline Provinces de la zone & Chef lieu & Superficie dans la zone \\
\hline Noms & Noms & $\mathrm{Km}^{2}$ \\
\hline Bazéga & Kombissiri & 5295,4 \\
Boulgou & Tenkodogo & 4743,5 \\
Boulkiémdé & Koudougou & 4070,4 \\
Ganzourougou & Zorgho & 4442,7 \\
Kadiogo & Ouagadougou & 418,8 \\
Kouritenga & Koupéla & 2347,2 \\
Nahouri & Po & 2222,2 \\
Namentenga & Boulsa & 2387,6 \\
Oubritenga & Ziniaré & 5286,8 \\
Passoré & Yako & 2076,0 \\
Sanguié & Réo & 2281,6 \\
Sanmatenga & Kaya & 2045,2 \\
Sissili & Léo & 12846,0 \\
Zoundwéogo & Manga & 3233,2 \\
\hline Parc Kaboré Tambi & & 1516,2 \\
\hline Ranch de Nazinga & & 987,2 \\
\hline Total de la zone & & 56200,0 \\
\hline
\end{tabular}

Source: (MET/PNUD/FAO,1990)

Cette zone concerne quatorze provinces qui sont partiellement ou entièrement inclues dans la zone délimitée. La zone délimitée couvre une superficie de 56200 kilomètres 
carrés et environ 60000 kilomètres carrés si la superficie totale de chaque province est considérée.

3.2. Évaluation du potentiel ligneux de la région de OuagadougouStratification des formations forestières et évaluation du potentiel ligneux

Premièrement, l'interprétation des images satellites Landsat TM a permis de définir les grandes limites des zones purement forestières par rapport à celles cultivées et de faire une première approche de stratification. Non seulement les différentes formations forestières et leur répartition dans l'espace ont été reconnues, mais leur densité à travers des taux de recouvrement végétatifs ont été établis en trois classes : Classe $1:$ plus de $60 \%$ de recouvrement. Classe 2 : de $20 \%$ à $60 \%$ de recouvrement et Classe $3:$ de $0 \%$ à $20 \%$ de recouvrement. Deuxièmement, des opérations de reconnaissance et de vérification sur le terrain ont été effectuées (dites de vérités de terrains) : ce qui a permis de confirmer ou de rectifier les données d'interprétation des images par satellite. Les travaux d'inventaire de terrain et de cartographie de la zone cible par l'équipe d'experts (IGB et FAO) a permis d'évaluer les stocks de bois sur pied disponible suivant trois strates exploitables des massifs forestiers caractérisés et identifiés dans la zone. Les coefficients de bois sur pied et les taux d'exploitation durable par strate sont donnés dans le tableau 4.

Tableau 4: Coefficients de bois sur pied et les taux annuels d'exploitation durable par strate

\begin{tabular}{|cccc|}
\hline Strates & Caractéristiques & $\begin{array}{c}\text { Quantité de } \\
\text { bois par } \\
\text { hectare }\end{array}$ & $\begin{array}{c}\text { Taux } \\
\text { d'exploitation } \\
\text { annuel }\end{array}$ \\
\hline Strate 1 & $\begin{array}{c}\mathrm{B}=\text { arboré à boisé } \\
\mathrm{T}=\text { bien arboré } \\
\mathrm{M}=\text { moyenne arboré }\end{array}$ & $\begin{array}{c}56 \text { stères par } \\
\text { hectares }\end{array}$ & $\begin{array}{c}\text { 2 stères par } \\
\text { hectares et par } \\
\text { an }\end{array}$ \\
\hline Strate 2 & $\begin{array}{c}\mathrm{A}=\text { arbustif à arboré } \\
\mathrm{R}=\text { relativement arboré }\end{array}$ & $\begin{array}{c}47 \text { stères par } \\
\text { hectares }\end{array}$ & $\begin{array}{c}\text { 1 stère par } \\
\text { hectares et par } \\
\text { an }\end{array}$ \\
\hline Strate 3 & $\begin{array}{c}\mathrm{F}=\text { faiblement arboré } \\
\mathrm{P}=\text { faiblement arbustif } \\
\mathrm{K}=\text { îlots de végétation } \\
\text { (terres cultivées, jachères) }\end{array}$ & $\begin{array}{c}36 \text { stères par } \\
\text { hectares }\end{array}$ & $\begin{array}{c}0,5 \text { stères par } \\
\text { hectares et par } \\
\text { an }\end{array}$ \\
\hline
\end{tabular}

Sources : Tableau établi à partir des interprétations photo - satellites dans les planimètres (1991)

Les chiffres d'évaluation des quantités de bois durablement exploitables annuellement retenus dans la zone sont faibles, et compris entre 0,5 et 2 stères par hectare et par an. Ces taux annuels d'exploitation durable par strate représentent les taux annuel d'accroissement naturel du bois sur pieds dans les différentes strates définies dans le tableau $n^{\circ} 4$, et sont calculés par le laboratoire de la FAO (1991) à Rome à partir des photos satellites et des vues aériennes effectuées par l'Institut Géographique du Burkina (IGB) sur les planimètres (ces chiffres représentent une mesure de ce que nous appelons « rendement maximum soutenable d'une forêt »).

Le concept d'exploitation durable est développé en économie des ressources naturelles et intègre les dimensions écologique, économique et sociale de l'existence de la ressource. La question de l'économie des ressources naturelles est de savoir comment traiter les actifs naturels environnementaux afin qu'ils puissent soutenir l'économie comme une source d'amélioration du niveau de vie (Pearce et Turner, 1990). Pour ces deux auteurs, 
les deux premières fonctions des ressources naturelles environnementales (fonction d'offre de ressources et d'assimilation de déchet) impliquent certaines règles de gestion des ressources naturelles si nous souhaitons maintenir ces fonctions pendant de longues périodes. Ces règles sont, d'abord, toujours utiliser les ressources renouvelables de telle sorte que le taux d'exploitation ne soit pas plus grand que le taux de régénération naturelle, et ensuite, toujours garder les flux de déchets jetés à l'environnement à un niveau inférieur à la capacité d'assimilation de celui-ci.

Une raison supplémentaire pour le maintien du stock de ressources est d'assurer un accès égal à ces ressources par différentes générations (Pearce et Turner, 1990, p. 43). Cette manière de penser a favorisé l'émergence d'outils de gestion et de partage inter-temporel des ressources naturelles. La principale caractéristique des ressources naturelles renouvelables est que leur stock n'est pas fixe et peut aussi bien augmenter que diminuer; il augmentera si le stock est capable de se régénérer. Néanmoins, il y a un stock maximum car aucune ressource renouvelable ne peut se reproduire au-delà des capacités qu'offre l'écosystème dans lequel elle existe. Toutes choses égales par ailleurs, il y a une raison de supposer que ce processus d'exploitation de la ressource peut se poursuivre pendant de très longues périodes. L'homme peut exploiter l'accroissement de la ressource, la laisser croître, puis prélever de nouveau ce supplément, et ainsi de suite, si certaines conditions sont réunies (Pearce et Turner, 1990, p. 241). Mais la surexploitation d'une ressource renouvelable est significative: il est très facile de faire disparaitre une telle ressource; c'est ce qui arrivera si le taux de prélèvement de la ressource excède le taux de régénération de celle-ci. La gestion forestière constitue l'autre grand domaine d'application de la théorie des ressources naturelles (Faucheux et Noël, 1995, p. 159). Plusieurs questions se sont posées aux économistes des ressources naturelles qui sont «la recherche du rendement maximum soutenable d'une forêt», "la détermination de la période de rotation optimale », et «l'impact de certaines variables économiques et environnementales sur la rentabilité et la période de rotation. » Ces questionnements vont favoriser le développement de modèles de gestion forestière par les économistes de ressources naturelles, les uns très complexes, les autres plus simples.

Le tableau 5 donne les superficies des massifs forestiers exploitables de la zone. 
Tableau 5 : Les superficies forestières exploitables des strates en hectares

\begin{tabular}{|c|c|c|c|c|}
\hline Provinces & Strate 1 & Strate 2 & Strate 3 & Total \\
\hline Bazéga & 16860 & 47720 & 120520 & 185100 \\
\hline Boulougou & 23290 & 93800 & 186780 & 303870 \\
\hline Boulkièmdé & 520 & 14300 & 96140 & 110960 \\
\hline Ganzourougou & 3000 & 46880 & 155030 & 204910 \\
\hline Kadiogo & 320 & 680 & 1680 & 2680 \\
\hline Kouritenga & 0 & 12580 & 66730 & 79310 \\
\hline Nahouri & 42180 & 59490 & 62430 & 164100 \\
\hline Namentenga & 0 & 9600 & 69240 & 78840 \\
\hline Oubritenga & 2000 & 11480 & 143800 & 157280 \\
\hline Passoré & 520 & 11520 & 72760 & 84800 \\
\hline Sanguié & 4160 & 20420 & 91400 & 115980 \\
\hline Sanmatenga & 1600 & 5880 & 84840 & 92320 \\
\hline Sissili & 303160 & 315160 & 216520 & 834840 \\
\hline Zoundwéogo & 40420 & 55980 & 79240 & 175640 \\
\hline Parc National Kaboré Tambi & 61160 & 68340 & 16500 & 146000 \\
\hline Ranch de Nazinga & 52500 & 41740 & 4080 & 98320 \\
\hline Total région de Ouagadougou & 551690 & 815570 & 1467690 & 2834950 \\
\hline Total hors parc et ranch & 438030 & 705490 & 1447110 & 2590630 \\
\hline
\end{tabular}

Source : Synthèse faite des planimètres de l'occupation des sols (1991)

Les superficies forestières potentiellement exploitables de la zone d'approvisionnement couvrent environ 2,6 millions d'hectares (celles concernant les parcs animaliers ayant été mis de côté). Ces superficies ne sont pas négligeables si elles sont exploitées dans un cadre réglementaire et de gestion économique durable. Mais encore faut-il évaluer le volume de bois sur pieds et hiérarchiser les provinces de la zone selon leurs potentialités forestières.

Le volume de bois durablement exploitable annuellement

34 Avec les coefficients d'exploitation durable (tableau 4), et les superficies exploitables fournies par ces planimètres (tableau 5), nous en arrivons aux volumes de bois durablement exploitables par province dans le tableau 6. 
Tableau 6 : Volume de bois durablement exploitable par an en stères

\begin{tabular}{lcccc}
\hline Provinces & Strate 1 & Strate 2 & Strate3 & Total \\
\hline Bazéga & 33720 & 47720 & 60260 & 141700 \\
Boulougou & 46580 & 93800 & 93390 & 233770 \\
Boulkièmdé & 1040 & 14300 & 48070 & 63410 \\
Ganzourougou & 6000 & 46880 & 77515 & 130395 \\
Kadiogo & 640 & 680 & 840 & 2160 \\
Kouritenga & 0 & 12580 & 33365 & 45945 \\
Nahouri & 84360 & 59490 & 31215 & 175065 \\
Namentenga & 0 & 9600 & 34620 & 44220 \\
Oubritenga & 4000 & 11480 & 71900 & 87380 \\
Passoré & 1040 & 11520 & 36380 & 48940 \\
Sanguié & 8320 & 20420 & 45700 & 74440 \\
Sanmatenga & 3200 & 5880 & 42420 & 51500 \\
Sissili & 606320 & 315160 & 108260 & 1029740 \\
Zoundwéogo & 80840 & 55980 & 39620 & 176440 \\
Parc National Kabpré & & & & \\
Tambi & 122320 & 68340 & 8250 & 198910 \\
Ranch de Nazinga & 105000 & 41740 & 2040 & 148780 \\
\hline Total & $\mathbf{1 1 0 3 3 8 0 8 1 5 5 7 0 7 3 3 8 4 5 2 6 5 2 7 9 5}$ \\
\hline
\end{tabular}

Source : Établi à partir des planimètres de l'occupation des sols (FAO/PNUD-BKF, 1991)

Cette évaluation tient compte de toutes les caractéristiques de l'occupation des sols dans la zone géographique telles que décrites et analysées dans les planimètres de l'occupation des sols de la région en 1991; ces planimètres du fait qu'ils ont été élaborés par des compétences spécialisées en la matière (F.A.O, IGB) et des moyens matériels et financiers qu'a nécessité leur mise en oeuvre, permettent d'avoir des résultats pertinents et fiables. Il ressort que le volume de bois durablement exploitable est de 2,66 millions de stères de bois par an soit 0,66 millions de tonnes de bois.

3.3. Population de la zone et ressources forestières

Le tableau 7 permet d'avoir l'ampleur de la population de la région d'approvisionnement de Ouagadougou en bois-énergie. 
Tableau 7: Population de la zone de ravitaillement de Ouagadougou en bois-énergie

\begin{tabular}{lcccccc}
\hline & \multicolumn{2}{c}{ Population 1975 } & \multicolumn{2}{c}{ Population 1985 } & \multicolumn{2}{c}{ Population 1996 } \\
\cline { 2 - 7 } Provinces & Taille & Proportion & Taille & Proportion & Taille & Proportion \\
\hline Bazéga & 217849 & $8,3 \%$ & 303941 & $8,1 \%$ & 398595 & $7,6 \%$ \\
Boulougou & 298491 & $11,4 \%$ & 402236 & $10,7 \%$ & 548928 & $10,4 \%$ \\
Boulkièmdé & 310989 & $11,8 \%$ & 365223 & $9,8 \%$ & 421302 & $8,0 \%$ \\
Ganzourougou & 124638 & $4,7 \%$ & 195652 & $5,2 \%$ & 256921 & $4,9 \%$ \\
Kadiogo & 194351 & $7,4 \%$ & 459826 & $12,3 \%$ & 941894 & $17,9 \%$ \\
Kouritenga & 106111 & $4,0 \%$ & 198486 & $5,3 \%$ & 444928 & $8,5 \%$ \\
Nahouri & 73485 & $2,8 \%$ & 105509 & $2,8 \%$ & 131472 & $2,5 \%$ \\
Namentenga & 168353 & $6,4 \%$ & 198890 & $5,3 \%$ & 226193 & $4,3 \%$ \\
Oubritenga & 242859 & $9,3 \%$ & 304265 & $8,1 \%$ & 346960 & $6,6 \%$ \\
Passoré & 218529 & $8,3 \%$ & 223830 & $6,0 \%$ & 271864 & $5,2 \%$ \\
Sanguié & 173442 & $6,6 \%$ & 217277 & $5,8 \%$ & 249583 & $4,8 \%$ \\
Sanmatenga & 283776 & $10,8 \%$ & 367724 & $9,8 \%$ & 464032 & $8,8 \%$ \\
Sissili & 120391 & $4,6 \%$ & 244919 & $6,5 \%$ & 353359 & $6,7 \%$ \\
Zoundwéogo & 91524 & $3,5 \%$ & 155777 & $4,2 \%$ & 197133 & $3,8 \%$ \\
Total & $\mathbf{2 6 2 4 7 8 8}$ & $100,0 \%$ & $\mathbf{3 7 4 3 5 5 5}$ & $100,0 \%$ & $\mathbf{5 2 5 3 1 6 4}$ & $100,0 \%$ \\
\hline
\end{tabular}

Source : Établi à partir des données « INSD, Recensements généraux décennaux 1975, 1985 et 1996. »

Les trois derniers recensements décennaux de 1975, de 1985 et 1996 vont nous permettre d'appréhender la population totale de la zone et les rythmes d'accroissement de celle-ci et aussi la concentration spatiale de celle-ci (la densité). La population comme nous l'avons déjà étudiée dans la revue de littérature est une variable déterminante dans la demande des ressources ligneuses et de façon générale dans la dégradation de la ressource (corrélation négative entre superficie forestière et densité de la population). C'est pourquoi, le rythme d'accroissement des défriches des superficies forestières peut s'appréhender à travers celui de la population (la densité de population). Les quatorze provinces qui concernent cette zone, regroupent une population de 5,25 millions d'habitants en 1996. La répartition de la population entre les provinces est très inégalitaire : celle du Kadiogo dont le chef lieu est la ville de Ouagadougou, se trouve non seulement être la plus petite $\left(418,8 \mathrm{~km}^{2}\right)$, mais aussi la plus peuplée avec une population de 0,942 millions d'habitants. Elle représente $17,9 \%$ de la population totale de la zone en 1996. Pour plus de précision, le tableau 8 donne les indices d'accroissement de la population de la zone et les densités de cette population par province pour les trois dates retenues. 
Tableau 8: Indices d'accroissement et densités de la population dans la zone.

\begin{tabular}{lcccccc}
\hline \multirow{2}{*}{ Provinces } & \multicolumn{3}{c}{ Taux d'accroissement de la population Densité de la population } \\
\cline { 2 - 7 } & \multicolumn{3}{c}{ Pourcentage (\%) } & \multicolumn{4}{c}{ Nombre d'habitants au km ${ }^{2}$} \\
\cline { 2 - 7 } & $1975-1985$ & $1985-1996$ & $\mathbf{1 9 7 5 - 1 9 9 6}$ & $\mathbf{1 9 7 5}$ & $\mathbf{1 9 8 5}$ & $\mathbf{1 9 9 6}$ \\
\hline Bazéga & 39,52 & 31,14 & 82,97 & 40,25 & 56,16 & 73,65 \\
Boulougou & 34,76 & 36,47 & 83,90 & 34,84 & 46,96 & 64,08 \\
Boulkièmdé & 17,44 & 15,35 & 35,47 & 73,81 & 86,68 & 99,99 \\
Ganzourougou & 56,98 & 31,32 & 106,13 & 29,27 & 45,95 & 60,34 \\
Kadiogo & 136,60 & 104,84 & 384,64 & 442,62 & 1047,22 & 2145,10 \\
Kouritenga & 87,06 & 124,16 & 319,30 & 41,81 & 78,21 & 175,32 \\
Nahouri & 43,58 & 24,61 & 78,91 & 20,49 & 29,42 & 36,66 \\
Namentenga & 18,14 & 13,73 & 34,36 & 24,74 & 29,22 & 33,23 \\
Oubritenga & 25,28 & 14,03 & 42,86 & 44,56 & 55,83 & 63,66 \\
Passoré & 2,43 & 21,46 & 24,41 & 58,03 & 59,44 & 72,20 \\
Sanguié & 25,27 & 14,87 & 43,90 & 33,34 & 41,77 & 47,98 \\
Sanmatenga & 29,58 & 26,19 & 63,52 & 30,65 & 39,72 & 50,12 \\
Sissili & 103,44 & 44,28 & 193,51 & 9,18 & 18,68 & 26,95 \\
Zoundwéogo & 70,20 & 26,55 & 115,39 & 25,05 & 42,63 & 53,94 \\
\hline Moyenne & $\mathbf{4 9 , 3 0}$ & $\mathbf{3 7 , 7 8}$ & $\mathbf{1 1 4 , 9 5}$ & $\mathbf{6 4 , 9 0}$ & $\mathbf{1 1 9 , 8 5}$ & $\mathbf{2 1 4 , 5 2}$ \\
\hline \hline
\end{tabular}

Source : Établi à partir des données « INSD, 1975, 1985 et 1996. » et « PNGT-BNDT,1996) de bois disponible exploitable et la densité de la population par province dans la zone de ravitaillement de Ouagadougou en bois-énergie: ce qui confirme l'impact de l'action anthropique sur la dégradation des ressources forestières. Cette corrélation montre que les provinces où la densité de la population est forte disposent le moins de ressources ligneuses. Cette moindre disponibilité relative de la ressource ligneuse est due aux demandes diverses (défriches culturales, besoins en bois de chauffage, surpâturages etc..). 4. L'évaluation de la demande globale de Ouagadougou en bois-énergie

Nous évaluons les coefficients de consommation par ménage et par artisan, les quantités consommées des ménages et des artisans en bois-énergie et leur consommation totale. Pour cela, il faut nécessairement disposer du nombre total des différents segments de consommateurs de cette énergie.

4.1. L'évaluation des coefficients de consommation par « la méthode de la conversion des dépenses »Evaluation des coefficients de consommation par habitant 
41 Le prix du marché du kilogramme de bois utilisé pour le calcul qui a été de 29,75 FCFA (INSD, 1996) est la moyenne des prix du bois en vigueur pendant les douze mois de 1996. Quant au prix du gaz, il a été de 312,5 FCA le kilogramme (Sonhaby, 1996). Les dépenses des ménages et ces prix permettent grâce à la méthode de la conversion des dépenses de passer aux quantités consommées de ces énergies par les ménages. La mise en oeuvre de cette méthode avec la base de données INSD-UEMOA (1996) donne les résultats suivants :

Tableau 9 : Coefficients de consommation des ménages en énergies de cuisson en 1996

\begin{tabular}{|l|c|c|c|}
\hline Types d'énergie & $\begin{array}{c}\text { Bois de } \\
\text { chauffe }\end{array}$ & $\begin{array}{c}\text { Charbon de } \\
\text { bois }\end{array}$ & Gaz butane \\
\hline $\begin{array}{l}\text { Coefficients en kg / } \\
\text { habitant / jour }\end{array}$ & 0,532 & 0,168 & 0,063 \\
\hline $\begin{array}{l}\text { Coefficients en FCFA/ } \\
\text { habitant / jour }\end{array}$ & 15,84 & 13,35 & 19,78 \\
\hline $\begin{array}{l}\text { Coefficients équivalent du charbon en } \\
\text { volume de bois }\end{array}$ & 0,839 & \\
\hline
\end{tabular}

Sources : Estimés à partir de la base de données INSD-PHPC/UEMOA (1996)

Selon le sondage que nous avons réalisé en août 1996, parmi les ménages utilisant le combustible ligneux comme source principale d'énergie de cuisson, $52 \%$ de ceux-ci possèdent un foyer amélioré. La promotion des foyers améliorés qui sont des foyers à haut rendement énergétique, ont contribué à réduire ce coefficient de consommation au cours des deux dernières décennies, le faisant passer de $1,18 \mathrm{~kg} /$ habitant / jour (De Backer, 1980) à $0,532 \mathrm{~kg} /$ habitant / jour en 1996. Certes, la raréfaction progressive de la ressource a induit également de nouvelles habitudes culinaires (par exemple, « la journée continue » qui consiste maintenant à faire la cuisson journalière des repas en une seule cuisson, le repas du soir devant être réchauffé) qui ont contribué à la réduction du coefficient de consommation.

De même pour évaluer la demande des artisans en bois-énergie, il faut pouvoir disposer des coefficients de consommation par jour et par artisan, les fréquences hebdomadaires de leur activité et de leur nombre dans l'environnement étudié. C'est pour toutes ces raisons que ces variables requises ont fait l'objet de questions dans les questionnaires administrés aux artisans.

Evaluation des coefficients de consommation par catégorie d'artisans

La méthode de la "conversion des dépenses» nous a permis dans ce cas également de procéder à l'évaluation des coefficients de consommation par catégorie d'artisans (confêre annexes tableau $n^{\circ} 1$ ). La connaissance des coefficients de consommation des différents segments de consommateurs de bois-énergie permet d'évaluer leur consommation annuelle.

4.2. Évaluation de la demande globale en bois-énergies de Ouagadougou en 2000

Les indicateurs de consommation vont maintenant permettre d'évaluer la consommation annuelle de ces catégories de demandeurs (ménages et artisans) du bois-énergie.

La consommation des ménages de Ouagadougou

La base de données utilisée ayant concerné les dépenses des ménages de l'année 1996, nous avons ainsi évalué directement la consommation effective de ceux-ci pour 1996 ( confere annexes tableau $n^{\circ} 2$ ). 
Sur la base d'un taux de croissance annuelle de la population de Ouagadougou de $6,2 \%$ au cours de la dernière décennie, nous pouvons évaluer la demande de cette ville en énergies de cuisson. Ainsi, nous obtenons la demande de la ville pour l'an 2000 (tableau $n^{\circ} 10$ ), donnant une demande ménagère de 95480,74 tonnes de feu, 2661,13 tonnes de charbon de bois, soit un volume total 108786,41 tonnes de bois-énergie

Tableau 10 : Évaluation de la demande ménagère des énergies de cuisson en l'an 2000

\begin{tabular}{|l|c|c|c|}
\hline & \multicolumn{2}{|c|}{ Nombre de ménages } & Consommation \\
\hline & Proportion & $\begin{array}{c}\text { Nombre de } \\
\text { personnes }\end{array}$ & $\begin{array}{c}\text { Annuelle en } \\
\text { tonnes }\end{array}$ \\
\hline Bois de chauffe & $70,10 \%$ & 624925 & 121455 \\
\hline Charbon de bois & $6,20 \%$ & 55271 & 3385 \\
\hline $\begin{array}{l}\text { Équivalent du Charbon de bois en volume de } \\
\text { bois de chauffe }\end{array}$ & 16925 \\
\hline $\begin{array}{l}\text { Demande totale du } \\
\text { bois-énergie }\end{array}$ & $76,3 \%$ & 680198 & 138380 \\
\hline Gaz butane & $13,0 \%$ & 115892 & 2677 \\
\hline
\end{tabular}

Sources: Estimés à partir de la base de données INSD-PHPC/UEMOA (1996)

De même, les ménages ont consommé 138380 tonnes de bois-énergie en 2000, et 2677 tonnes de gaz butane pour les ménages.

La demande artisanale de l'an 2000

Puisque les coefficients de consommation des artisans sont fonction de l'intensité de leur activité, l'évaluation de leur demande nécessite la connaissance des fréquences hebdomadaires de l'activité de ces catégories d'artisans, saisies par les enquêtes et synthétisées en annexes tableau $n^{\circ} 3$ (Confère annexes tableau $n^{\circ} 2$ ). Les dolotières qui ont le coefficient ${ }^{2}$ de consommation le plus importante présentent la fréquence de cuisson la plus faible, d'une moyenne hebdomadaire des préparations de 2 ; mais cette fréquence est à relativisés puisque la cuisson du dolo est continue et s'étend sur 8 à 10 heures par jours et pendant deux jours consécutifs.

Le nombre des artisans a pu être estimé grâce aux enquêtes sur les artisans en mai 2000. En utilisant la variable durée de l'activité des artisans dans les sondages que nous avons, nous avons cumulé les fréquences des artisans ayant commencé leur activité il y a au plus 10 ans et nous avons utilisé ces proportions comme représentant les artisans de la catégorie qui sont rentrés dans le secteur d'activité concerné au cours de la dernière décennie. Ces proportions sont de $25 \%$ pour les dolotières, de $81 \%$ pour les grilleurs de viande et de $75 \%$ pour les restaurateurs. Cette méthode nous a permis d'évaluer de manière beaucoup plus efficace et réaliste le nombre de ces dernières catégories d'artisans. Ce qui par conséquent a facilité l'évaluation de la demande de bois-énergie des artisans pour l'année 2000 dans le tableau 11: 
Tableau 11 : Demande des catégories d'artisans en bois-énergie en l'an 2000

\begin{tabular}{|l|c|c|c|c|c|c|}
\hline \multicolumn{7}{|c|}{$\begin{array}{c}\text { Nombre Fréquence Coefficient de Demande } \\
\text { d'artisans annuelle consommation 2000 (kg) des artisans }\end{array}$} \\
\hline Artisans/ Années & $\mathbf{1 9 9 0 ^ { \mathbf { a } }}$ & $\mathbf{2 0 0 0}$ & $\mathbf{2 0 0 0}$ & Journalier & Annuel & Tonnes \\
\hline Fondeurs de bronze & 20 & 231 & 365 & 86,49 & 31568,85 & 7292,404 \\
\hline Fondeurs d'Aluminium & 36 & 240 & 312 & 181,40 & 56596,80 & 13583,232 \\
\hline " Dolotières " & 760 & 1013 & 104 & 458,17 & 47649,68 & 48269,126 \\
\hline Grilleurs de viande & 516 & 2715 & 365 & 60,63 & 22131,14 & 60086,058 \\
\hline Restaurateurs & 524 & 2096 & 365 & 57,10 & 20842,76 & 43686,423 \\
\hline $\begin{array}{l}\text { Demande artisanale totale de bois-énergie à Ouagadougou pour l'an } \\
\text { 2000 }\end{array}$ & 171858,346 \\
\hline
\end{tabular}

Source: a =INSD (1991) ; Analyse statistique des données d'enquêtes sur les artisans (mai, 2000)

Chaque préparation dure deux jours de cuisson pour les dolotières, c'est ce qui explique une moyenne de consommation de bois par préparation de près d'une demie tonne (458,17 kilogrammes de bois de feu) en moyenne. Cependant, il faut noter qu'il existe une forte dispersion au niveau des fréquences de préparation hebdomadaires, qui, pour les unes est d'une préparation pour toutes les deux semaines, qui, pour certaines est d'une fois par semaine, qui pour d'autres est de deux fois par semaine et enfin pour une dernière catégorie qui arrivent à produire trois fois par semaine. Les dolotières qui produisent moins de deux fois par semaine, commercialisent elles-mêmes leur production, tandis que celles qui produisent au moins deux fois par semaine, le font pour les revendeuses pour la plus grande partie de leur production et commercialisent par elles-mêmes seulement une petite partie de celle-ci. Ainsi, l'évaluation de la demande artisanale de bois-énergie pour l'an 2000 des artisans ciblés par le sondage, donne un chiffre d'environ 172000 tonnes de bois-énergie. Aussi, l'évaluation conjointe de la demande des ménages et des artisans de Ouagadougou permet enfin l'évaluation de la demande globale de cette ville.

La consommation globale en bois-énergie

Une hypothèse supplémentaire doit être postulée si nous ne voulons pas sous-évaluer la consommation globale de cette ville en bois-énergie, puisque l'enquête sur la consommation des artisans n'a pas pu couvrir toutes les catégories d'artisans qui utilisent le bois-énergie comme facteurs de production. C'est le cas des bijoutiers, des potiers, des vendeurs de café, des kiosques de restauration. C'est dans ces conditions que nous formulons l'hypothèse faible que : "la consommation de toutes ces autres catégories d'artisans représente $10 \%$ de la consommation totale des ménages et des artisans ciblés par les enquêtes de mai 2000.» Cette contribution de 10\% des autres artisans non ciblés dans la collecte se justifie par leur important nombre dont le dénombrement aura exigé un recensement exhaustif aux coûts éventuels très onéreux, et surtout l'ampleur de leur coefficient de consommation journalière par type d'artisan dont le poids est donné par le tableau 12. 
Tableau 12 : Indicateurs de consommation d'autres types d'artisans en charbon de bois

\begin{tabular}{|l|c|c|c|c|c|}
\hline $\begin{array}{l}\text { Types } \\
\text { d'artisans }\end{array}$ & $\begin{array}{c}\text { Coefficients de } \\
\text { consommation }\end{array}$ & $\begin{array}{c}\text { Écart } \\
\text { type }\end{array}$ & $\begin{array}{c}\text { Nombre } \\
\text { d'observations }\end{array}$ & $\begin{array}{c}\text { Durée des } \\
\text { pesées } \\
\text { 3 jours }\end{array}$ & $\begin{array}{c}\text { Année de } \\
\text { sondage }\end{array}$ \\
\hline Tailleur & 0,708 & 0,278 & 10 & Mars 2000 \\
\hline Blanchisseurs & 1,005 & 0,318 & 10 & 3 jours & Mars 2000 \\
\hline $\begin{array}{l}\text { Buveurs de } \\
\text { Thé }\end{array}$ & 1,896 & 1,171 & 10 & 3 jours & Mars 2000 \\
\hline Cafetiers & 3,275 & 2,527 & 10 & 3 jours & Mars 2000 \\
\hline Kiosques & 3,016 & 2,527 & 4 & 3 jours & Aoât 1999 \\
\hline
\end{tabular}

${ }^{*} \mathrm{Ce}$ coefficient est donné en kilogramme par artisan et par jour. Le taux de $10 \%$ est à notre sens minimal car ces artisans sont très nombreux dans la ville de Ouagadougou. II aurait donc fallu un dénombrement exhaustif, donc très onéreux pour pouvoir quantifier leur demande. Ce qui donne tout de même demande artisanale quelque peu sous évaluée.

Sources : APEX/RPTES (2000, p. 27); Edene (1999)

Ces artisans sont en nombre très important et l'inexistence de statistiques fiables sur leur effectif a contraint fortement l'évaluation de leur consommation, nous amenant à formuler une hypothèse basse de leur contribution à $10 \%$ de la consommation totale de Ouagadougou: ce qui nous permet de prendre en compte leur demande. Ainsi, nous arrivons à évaluer pour l'an 2000, la demande globale de la ville de Ouagadougou en boisénergie dans le tableau 13.

Tableau 13: Demande globale de Ouagadougou en bois-énergie pour l'an 2000

\begin{tabular}{|l|c|c|}
\hline $\begin{array}{l}\text { Types de } \\
\text { consommateurs }\end{array}$ & $\begin{array}{c}\text { Quantités } \\
\text { consommées } \\
\text { en tonnes de bois- } \\
\text { énergie }\end{array}$ & $\begin{array}{c}\text { Proportion } \\
\text { consommée par } \\
\text { type de } \\
\text { consommateurs }\end{array}$ \\
\hline Ménages & 138379,803 & $41 \%$ \\
\hline Artisans ciblés & 171858,346 & $50 \%$ \\
\hline Autres artisans & 31023,815 & $9 \%$ \\
\hline $\begin{array}{l}\text { Consommation } \\
\text { totale }\end{array}$ & $\mathbf{3 4 1 2 6 1 , 9 6 4}$ & $\mathbf{1 0 0 \%}$ \\
\hline
\end{tabular}

Source : Évaluation résultant des enquêtes INSD-PHPC/UEMOA (1996) et (mai, 2000).

Ce tableau montre que la capitale est très fortement dépendante de l'énergie ligneuse qui reste la principale source d'énergie de cuisson pour les ménages (dont 76,3\%) et les artisans (dont la presque totalité). La demande globale pour l'an 2000 qui se chiffre à 341262 tonnes de bois-énergie se partage en proportion de $41 \%$ pour les ménages, et en proportion de $59 \%$ pour les artisans. La promotion de l'artisanat et le gonflement de ce secteur qui devient de plus en plus important, laisse prévoir une amplification de cette demande avec pour conséquence une accélération des coupes de la ressource ligneuse dans la région d'approvisionnement. Ainsi, les artisans, malgré leur petit nombre par rapport au nombre de ménages, sont les plus grands utilisateurs du bois-énergie, surtout à cause du caractère pécuniaire et économique de leurs activités. L'évaluation de la consommation globale de la ville en bois-énergie peut permettre d'appréhender l'impact de la demande de la capitale en bois de feu sur le patrimoine forestier de la région et du 
pays par une analyse du décalage entre la consommation effective et l'offre potentielle durable de la zone d'approvisionnement.

5. Impact de la demande de Ouagadougou sur le potentiel forestier de la zone

Dans cette section, nous évaluons l'impact de la seule demande de Ouagadougou en boisénergie sur le patrimoine forestier de la zone de ravitaillement. Cependant le recoupement des quantités offertes qui est basé sur la production des chantiers aménagés et encadrés par les projets forestiers, et sur les enquêtes « des flux d'entrée » sous-estime la consommation de la ville en bois-énergie. C'est pourquoi nous privilégions le recoupement des quantités consommées effectives du bois-énergie pour évaluer l'impact de celles-ci sur les ressources ligneuses de la zone de ravitaillement de cette cité. Cette analyse se fait en comparant l'offre potentielle durable de la région d'approvisionnement et la consommation effective de Ouagadougou. Les superficies boisées ont connu une réduction moyenne de $58 \%$ entre 1980 et 1995 , avec un taux modal de réduction de $66 \%$ dans la région du Centre qui abrite la capitale (M.E.F. - Revue des Dépenses Publiques, 1996). Nous avons utilisé un inventaire forestier de 1990 de la zone d'approvisionnement de cette ville, réalisé par le laboratoire de la F.A.O. à Rome en collaboration avec l'Institut Géographique du Burkina (I.G.B.). Du fait que l'évaluation de l'impact porte sur l'année 2000, il était nécessaire de connaitre le volume de bois durablement exploitable en 2000. Aussi, nous maintenons l'hypothèse moyenne que "le taux de réduction des superficies boisées entre 1990 et 2000 dans cette zone reste égal à la moyenne nationale dans une moindre amplitude soit les $2 / 3$ de $58 \%$ ", malgré la hausse progressive des superficies de forêts naturelles aménagées, celles cultivées et la demande de l'énergie ligneuse. Ce taux sera donc de $38,67 \%$ pour les dix dernières années. Cette hypothèse va permettre de faire une analyse de l'impact de la demande Ouagadougou en bois-énergie sur le patrimoine forestier de sa région d'approvisionnement dans le tableau 14.

Tableau 14: Impact de la demande de Ouagadougou sur le patrimoine forestier.

\begin{tabular}{|l|c|c|}
\hline $\begin{array}{l}\text { Volume durable de bois } \\
\text { exploitable }\end{array}$ & Quantité en stères & $\begin{array}{c}\text { Demande/ } \\
\text { Volume }\end{array}$ \\
\hline Volume total durable en 1990 & 2652795 & $52 \%$ \\
\hline Volume hors ranch en 1990 & 2305105 & $60 \%$ \\
\hline Volume total durable en 2000 & 1626959 & $85 \%$ \\
\hline Volume hors ranch en 2000 & 1413721 & $97 \%$ \\
\hline $\begin{array}{l}\text { Demande totale en 2000 } \\
\text { Superficies défrichées en hectares en 2000 pour la } \\
\text { demande de la ville }\end{array}$ & 29699,057 \\
\hline $\begin{array}{l}\text { Superficie de Ouagadougou en hectares } \\
\text { Rapport superficies défrichées sur superficie de } \\
\text { Ouagadougou }\end{array}$ & 62500 \\
\hline
\end{tabular}

Sources : MEE/BKF/PNUD/FAO (I990) ; Enquêtes (mai, 2000) ; INSD-UEMOA/IHPC (1996).

Ainsi, la ville de Ouagadougou a consommé $85 \%$ du volume total de bois durablement exploitable de la zone d'approvisionnement qui compte quatorze provinces regroupant quelques grandes villes importantes du pays telles que Koudougou, Tenkodogo, Zorgho, Koupéla, Pô, Boulsa, Ziniaré, Yako, Réo, Kaya, Léo et Manga. Ces villes sont d’autant plus 
importantes qu'elles consomment également beaucoup de bois-énergie. Par exemple, certaines de ces villes sont de très grandes productrices de bière de sorgho rouge «le dolo » : c'est le cas de Réo, de Manga. Il faut noter que la province du Zoundwéogo dont le chef lieu est Manga est spécialisée dans la production du sorgho rouge, qui est la matière première principale dans la production du « dolo ", la bière de sorgho rouge. À cet effet, elle constitue la principale province d'approvisionnement de la capitale en sorgho rouge. Dès 1993, on dénombrait en milieu rural de ce pays 253505 « dolotières » dont 1573 dans la province du Kadiogo (ENSA, 1998) : ce chiffre est très important à souligner à cause de la valeur élevée du coefficient de consommation par dolotière et par préparation.

En excluant les superficies forestières des ranches, cette ville aurait consommé $97 \%$ du volume total de bois durablement exploitable dans la région de Ouagadougou: ce qui veut dire que les $3 \%$ du volume durablement exploitable devraient être exclusivement consacrés à la consommation de toutes les autres villes de la région de ravitaillement si on ne veut pas entamer le stock de ressources forestières reproductible. En considérant le coefficient moyen de bois sur pieds dans cette zone qui est de 46,33 stères de bois par hectare, la ville a défriché en 2000, 29699 hectares de bois-énergie pour sa consommation soit en proportion environ la moitié de sa propre superficie. Cette superficie représente $1,05 \%$ des superficies boisées des strates 1,2 et 3 de la région de ravitaillement de Ouagadougou en bois-énergie.

La demande en énergie ligneuse n'est pas la seule force motrice de la dégradation des ressources forestières dans la région. En effet, la forte densité de la population avec une corrélation négative de l'ordre de $-22 \%$ avec la disponibilité de ces ressources dans les quatorze provinces que regroupe la région (voire annexes tableau $\mathrm{n}^{\circ} 4$ pour le coefficient de corrélation de Pearson et le test d'indépendance), est une source de demande de nature diverse de ces ressources ligneuses tels le bois de service, le bois d'?uvre d'art, les fourrages pour les animaux, et les racines et écorces pour la pharmacopée. En plus, la région de ravitaillement qui concerne les régions administratives du Centre, du Centre Nord et du Centre Ouest du pays, connait depuis 1985 une moyenne de la densité de l'élevage de 16,67 Unités Bovines Tropicales par kilomètre carré contre une capacité de charge moyenne de l'ordre de 14,17 Unités Bovines Tropicales par kilomètre carré (PNFV, MARA, MEE, INSD; 1985) : ce qui veut dire que l'élevage extensif exerce également une pression non négligeable sur les ressources forestières. Enfin, les pratiques culturales dans la zone sont très néfastes avec une occupation des sols par les cultures et jachères de l'ordre de 50\% à 60\% (FAO/BKF/MEE, 1990).

59 L'ensemble de ces facteurs moteurs de la demande des ressources forestières fait que cette région est fortement menacée par la désertification. L'analyse statistique des études prospectives réalisées sur la demande de la ville en bois-énergie au cours des deux dernières décennies a très souvent soit surestimée ou sous-estimée cette demande. D'une part, à cause d'une utilisation de coefficients moyens de consommation par tête d'habitant et/ou par artisan qui ne résument pas la réalité du fait de la variation saisonnière de la demande et de l'offre de la ressource ligneuse, et du choix des périodes d'enquête devant conduire à l'évaluation de ces coefficients. D'autre part, à cause de la non-prise en compte de certaines catégories d'artisans et de la non connaissance de leur nombre effectif.

60 Nous avons aussi dans le cadre de cette réflexion pu corriger certains problèmes d'évaluation. D'une part, nous avons introduit la méthode de " conversion des dépenses " avec l'utilisation conjointe des prix moyens annuels du kilogramme de bois-énergie 
donnés par l'INSD, afin d'évaluer la demande globale de Ouagadougou, et de reconstituer le coefficient de consommation par tête d'habitant des utilisateurs potentiels du boisénergie. D'autre part, nous avons pu corriger le coefficient de consommation par dolotière qui était donné par jour, en le rétablissant par préparation et par dolotière et en utilisant la fréquence moyenne hebdomadaire de préparations des dolotières pour arriver à des évaluations plus réelles de la demande de cette catégorie d'artisans. En outre, une revue des annuaires statistiques de l'INSD sur les recensements des artisans au cours de la dernière décennie nous a permis à l'aide de la variable «durée de l'activité » introduite dans le questionnaire des artisans, d'appréhender les proportions des artisans qui sont entrés dans le secteur d'activités concernées au cours des années 90. Cette forte dégradation des ressources forestières constatée aujourd'hui autour de Ouagadougou éprouvent les politiques mises en ?uvres, et des questions se posent toujours sur les causes de ces échecs.

6. Esquisse d'évaluation des échecs des politiques de prix en amont de la filière

Malgré la mise en ?uvre d'une réglementation et d'une taxation (écotaxes) de l'exploitation de l'énergie ligneuse, la production frauduleuse issue des zones de production non encadrées (non organisées) reste très importante et les prix du boisénergie restent toujours à un niveau bas, très peu incitatif à l'émergence d'une filière privée de plantation d'arbres à but énergétique et à l'adoption de substituts à cette ressource ligneuse. Cette filière est celle qui exploite le bois dans les zones non aménagées.

6.1. Une importante production frauduleuse du bois-énergie

62 Avec une demande globale en bois-énergie de1,38 millions de stères de bois en 2000, seulement $15 \%$ de la demande de Ouagadougou proviennent des chantiers aménagés et/ ou contrôlés par les services forestiers: ce qui signifie que, $85 \%$ de cette demande proviennent des producteurs indépendants et occasionnels du bois-énergie (confère annexes tableau $\left.{ }^{\circ} 5\right)$. Ce même taux de $15 \%$ de production inventoriée avait été déjà trouvé (Ouédraogo, 1996), et un taux de 17\% obtenu par Pascale Delnooz (1999). Tous les $85 \%$ de cette demande passent outre la réglementation en vigueur: dès lors, les moyens de contrôle devraient être orientés vers cette production non inventoriée ou frauduleuse, si l'on veut décélérer la déforestation dans le pays et contrôler les flux d'entrées de ce combustible dans les centres de grande consommation. Les exploitants concernés par cette production sont pour la plupart des paysans en quête de revenus monétaires, utilisant des moyens de transport très modestes pour perpétuer cette activité de cueillette.

Une politique des prix ne peut être opérationnelle si les exploitants concernés par cette production restent en marge de la réglementation et des autres instruments de régulation de la filière bois-énergie. De même, une application effective des instruments économiques de régulation et de contrôle de la filière sur cette production procurerait suffisamment de ressources financières à l'administration publique pour la gestion durable de la ressource. Environ 1,051 milliards de FCFA n'ont pu être couverts du fait de la non application complète et effective des instruments de contrôle et régulation de la filière bois-énergie dans la région de Ouagadougou (confère annexes tableau $\left.n^{\circ} 6\right)$. Ce chiffre est sous-estimé si on considère la consommation totale des autres centres urbains de cette région. Tout indique alors que la mobilisation des moyens humains est insuffisante pour assurer l'application effective des politiques de prix. Le rapport entre surfaces aménageables de cette zone et le nombre total des agents exécutants va servir 
d'indicateur pour juger de la faiblesse des moyens humains mis en ?uvre pour faire appliquer les instruments de politique forestière définis. En effet dans l'aire d'approvisionnement de cette ville en bois de chauffe, les quatorze provinces concernées regroupent ensemble 120 agents de l'Inspection Générale des Eaux et Forêts, toutes catégories confondues dont $55 \%$ d'exécutants, $30 \%$ de cadres moyens et $15 \%$ de cadres supérieurs. En ne considérant que les $13 \%$ de forêts économiquement aménageables de la zone d'approvisionnement de cette cité, soit 700000 hectares, la couverture de contrôle est de 10606 hectares par agent forestier de cette zone. Ce qui paraît surestimé au regard du nombre d'agents de bureaux qui sont contraints de rester dans les centres. D'ailleurs, les contrôles sont irréguliers et aléatoires car les sorties de contrôle sont rares et quelquefois très espacées les unes des autres. Pour atteindre une couverture de contrôle de 500 hectares par agent forestier, il faudrait augmenter le nombre d'agents forestiers de cette zone de 1400 agents, soit un accroissement par rapport au potentiel humain actuel disponible de 1066,7\% (Ouédraogo, 1996). Comme la réalisation de tels investissements paraît onéreuse, les conditions minimales de gestion du patrimoine forestier seraient, comme précédemment signalé, la création de postes de police forestière aux différentes voies d'accès de la ville pour saisir et taxer les entrées de bois-énergie.

6.2. La production frauduleuse: une explication des échecs des politiques

L'importance de la production incontrôlée ou frauduleuse qui échappe aux taxes et redevances forestières détourne les politiques de prix et l'émergence des plantations privées d'arbres à but énergétique. La figure 1 permet de rendre compte cette réalité.

Figure 1: Effet de la production frauduleuse sur le niveau de prix du marché.

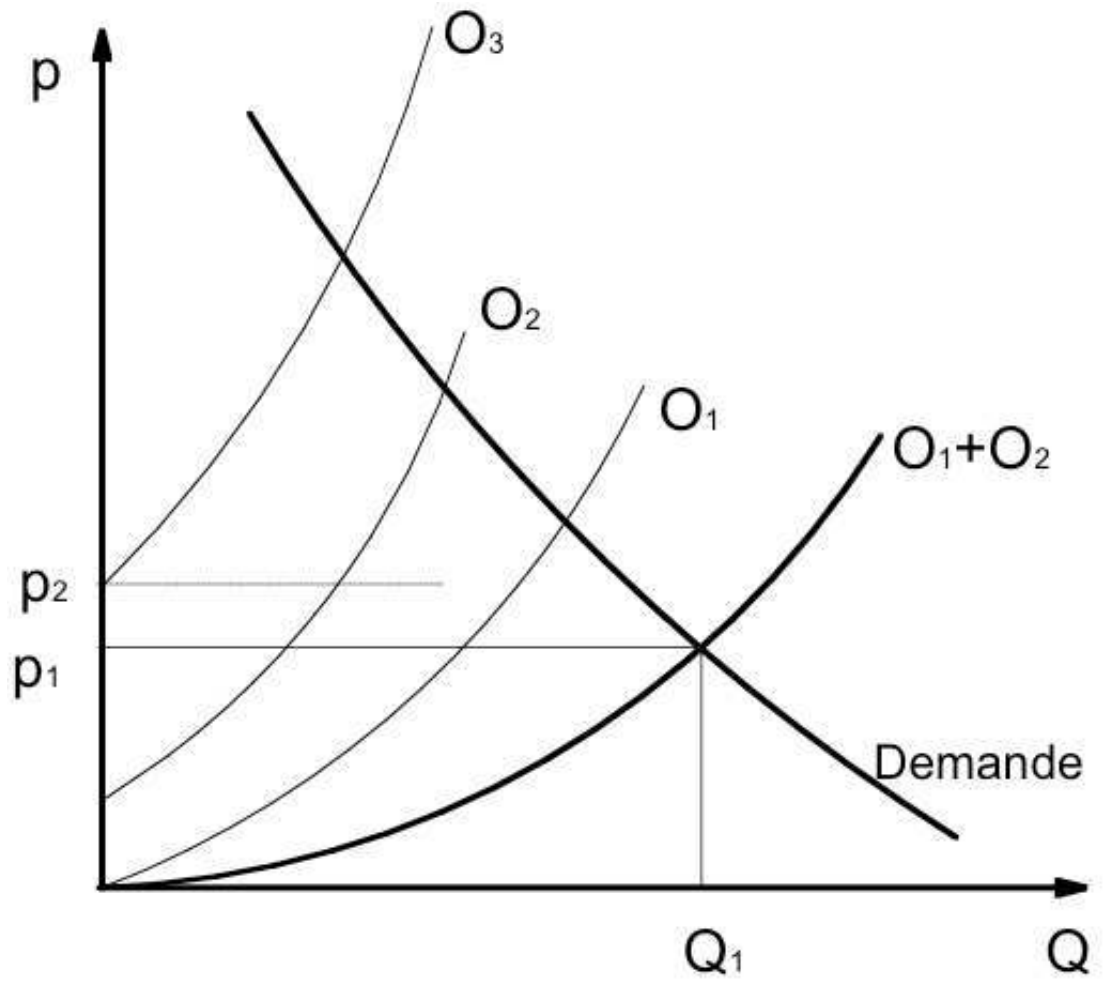

On désigne par $\mathrm{O}_{1}$ l'offre de la filière qui échappe aux éco-taxes (taxes et redevances forestières, fonds de roulement) et par $\mathrm{O}_{2}$ l'offre qui acquitte les éco-taxes et s'approvisionne sur les projets forestiers. La courbe $\mathrm{O}_{1}+\mathrm{O}_{2}$ est l'agrégation de ces deux 
offres. Compte tenu de la demande, on a une situation d'équilibre $p_{1}, Q_{1}$. La courbe $O_{3}$ correspond à l'offre encore assez virtuelle d'opérateurs s'appuyant sur des projets privés de replantation à proximité du centre de consommation. Au prix pratiqué $\mathrm{p}_{1}$, l'offre potentielle $\mathrm{O}_{3}$ ne peut se manifester. Il faudrait dépasser le niveau de prix $\mathrm{p}_{2}$ pour que cette offre se manifeste et participe à l'approvisionnement de Ouagadougou. On se maintient au prix $\mathrm{p}_{1}$ parce que l'offre $\mathrm{O}_{1}$ tire le prix vers le bas. Cette situation résulte d'un contrôle insuffisant de la part de la puissance publique. Cela crée une situation de prix artificiellement bas qui décourage les recherches de substitut et interdit à une filière s'appuyant sur une reforestation privée de voir le jour.

L'examen des causes des échecs des politiques de prix révèle que l'avenir de la filière boisénergie repose sur l'émergence d'une filière qui s'appuie sur une reforestation privée. Malgré les hausses respectives des prix au producteur du bois-énergie en 1995 et 1998, l'importance de la production frauduleuse tire le prix d'équilibre vers le bas, à des niveaux très peu incitatifs pour favoriser l'émergence d'une reforestation privée et la réponse des politiques de substitution par des sources d'énergie plus propres. Il apparaitt nécessaire d'élever le prix du bois de feu jusqu'au prix P2 de la figure $\mathrm{n}^{\circ} 1$. Cette hausse des prix passe nécessairement par un contrôle effectif de la production du bois-énergie dans la zone de ravitaillement de Ouagadougou, et donc un regain d'attention des pouvoirs publics dans la gestion de la filière. Un contrôle effectif de la production du boisénergie est une condition nécessaire pour l'émergence de planteurs privés d'arbres à but énergétique et pour une réponse positive des politiques de prix.

Conclusion

La filière bois-énergie à Ouagadougou constitue un important marché d'un chiffre d'affaires global de 5,8 milliards de FCFA en 2000. Cette filière concerne près de 50000 débiteurs, quelques projets forestiers, 10000 artisans, et 1000000 consommateurs potentiels en l'an 2000. La demande globale de Ouagadougou en bois-énergie a été évaluée à 341262 tonnes en 2000, soit environ 1,38 millions de stères, avec une part de 59\% comme consommation intermédiaire pour les artisans et $41 \%$ au titre de la consommation des ménages. A partir de 2000, cette demande contribuera à défricher (mettre complètement à nu) annuellement environ 30 milles hectares de forêts d'une densité moyenne de 46,33 stères de bois par hectare. Cette superficie représente 1,05\% des superficies boisées de la région de ravitaillement de Ouagadougou en bois-énergie, soit la moitié de la superficie de cette ville. Cette réalité nous fait dire de Ouagadougou qu'elle est la «brûleuse des forêts» au Burkina Faso. Ainsi, l'ampleur des effets de la demande de Ouagadougou en bois-énergie sur la dégradation du patrimoine forestier confirme l'hypothèse selon laquelle « la demande en énergie ligneuse constitue la principale cause de l'épuisement des ressources ligneuses dans la zone de ravitaillement de la capitale. » Cependant, l'analyse globale des forces motrices de la dégradation des ressources forestières dans la zone de ravitaillement de Ouagadougou a montré que d'autres facteurs tels que l'élevage extensif, l'agriculture extensive, la demande en bois de service et les baisses tendancielles de la pluviosité exercent une pression considérable sur les ressources forestières.

Malgré l'ampleur et la diversité des politiques pour rationaliser la consommation du boisénergie et pour le substituer par d'autres énergies alternatives plus écologiques, et celles d'aménagement des forêts naturelles pour l'approvisionnement des populations en boisénergie, la dégradation de la ressource ligneuse continue à un rythme inégalé, et l'impact 
de ces politiques reste négligeable. L'ensemble de ces politiques a coûté des dizaines de milliards de francs CFA au pays et à ses partenaires au développement. Tout se passe comme s'il y avait une inertie absolue des comportements de demande des énergies de chauffage, une inertie qu'il faudrait chercher à expliquer afin de contribuer à une plus grande efficacité de ces politiques. Beaucoup d'encre a coulé, beaucoup de séminaires et d'ateliers ont été tenus, et des lois ont été votées sur ce problème de la dégradation des ressources forestières; mais pourquoi les comportements d'offre et demande ne changent-ils pas malgré les politiques? Est-ce une incapacité de l'Etat à faire appliquer les lois qu'il fait voter lui-même? Est-ce une démagogie politique, ou un manque de moyens financiers?

\section{BIBLIOGRAPHIE}

Anderson Dennis (1996), « Energie et environnement: possibilités techniques et économiques », Revue Finances \& Développement, Paris.

Bationo B. Claude (1993), «Consommation et production de combustibles ligneux au Burkina Faso: analyse prospective et situation actuelle », Mémoire de D.E.A. FASEG/UNIVERSITE DE OUAGADOUGOU.

Beremba Francis, (1992), « Prix de Revient du Bois et Rentabilité Economique du Projet BKF/89/011/FOBF/89/011», Ouagadougou.

Chavin H., (1981), Une Ville Africaine en Crise d'Énergie: Ouagadougou, Edition Unasylva.

Cochet Hubert (1993), « Agriculture sur brûlis, élevage extensif et dégradation de l'environnement en Amérique Latine », Revue Tiers Monde, XXXIV(134).

Compaore A., (1993), «Atelier sur l'approvisionnement de Ouagadougou en Bois-énergie : La Solution est-elle Viable?», Document de guide de réflexion portant sur la maîtrise de la ressource Ouagadougou.

Coopération technique Allemande (Dietrich-Steve Sepp), (1986) « Réflexion sur l'arbre et les formations ligneuses au sahel Burkinabè », GTZ, Ouagadougou.

De Backer, M., Massart, L., Thombiano, T., 1981. « Développement des ressources forestières et renforcement du service forestier. Le ravitaillement des centres urbains ", Résultats provisoires, Notes de travail $n^{\circ} 8$.

Delnooz Pascal, (1999), « Gestion des Ressources Forestières : la Communauté, l'Etat et le Marché. Etude de Projets au Burkina Faso. » Fondation Universitaires Luxembourgeoise ; Thèse de Doctorat en Sciences de l'Environnement ; Tomes $1 \& 2,545$ pages.

Enda-Iepe, (1995), «L'Energie en Afrique : Situation Énergétique dans 34 Pays. », Enda Tiers Monde, Dakar.

ESMAP, (1991), «Burkina Faso: stratégie pour l'énergie ménagère », rapport n 134/91, Ouagadougou.

FAO (1993), «Analyse du secteur bois-énergie au Burkina Faso »ugou, FAO, Ouagado. 
FAO (1984), «Analyse statistiques de la consommation de bois de feu au Burkina faso », FAO, Rome. FAO (1985), «Organisation de l'exploitation forestière: cas du ravitaillement de la ville de Ouagadougou en bois de chauffe », FAO, Rome.

FAO (Novembre 1987), «Etude sur la contribution du secteur forestier à l'économie du Burkina Faso », FAO, Rome.

FAO (1995), «Évaluation des ressources forestières 1990 » , n¹12, Rome.

FAO (1996), «Le Burkina Faso : Document de Politique Forestière Nationale », Programme de coopération technique, Rome.

Ferari Victor (1990) « Du bois de chauffe au gaz butane: Ouagadougou et Bobo », Rjiksuniversiteit Groningen, Pays Bas.

Faucheurx S., Noël J.-F., (1995), Economie des ressources naturelles et de l'environnement ", Armand Colin, Paris.

Fontes J., Guinko S., (1995), « Cartes de la Végétation et de l'Occupation du Sol , Burkina Faso », ICIV, UMR9964/CNRST, IDR, FAST, IRBET, MET : Ouagadougou.

GFIG/GTZ, (1995), « Rapport sur l'atelier d'évaluation et programmation des activités du projet », Ouagadougou.

IBE (YAMEOGO Gabriel, 1992), «Bilan énergétique du Burkina Faso: 1970-1990 », Ouagadougou: DFVAF.

NSD,(1998); « Analyse des Résultats de l'Enquête sur les Dépenses des ménages de Ouagadougou » ; MEF, Ouagadougou.

Kabore Frédéric, (2000), « La demande de bois de feu comme facteur de production : cas des dolotières de la ville de Ouagadougou », FASEG : Mémoire de DEA, Université de Ouagadougou.

MEE (1996), « Quelles Réglementations Pour l'Exploitation des Produits Forestiers? »,

MEE, (1996), «Atelier national sur l'exploitation du bois: quelle réglementation pour l'exploitation des produits forestiers», Ouagadougou.

MEF, (1996), «Revue des Dépenses Publiques du Burkina Faso», Ouagadougou.

MEF, (1998), «Rapport sur l'Economie du Burkina Faso», Ouagadougou.

MET (OUEDRAOGO Felix, 1988), « Recueil de Textes Réglementant l'Exploitation du Bois: du 4 Juillet 1935 à nos Jours .», Ministère de l'Environnement et du Tourisme: Ouagadougou.

MET (1988), « Recueil de textes portant sur l'exploitation du bois (du 4 Juillet 1935 à nos jours », Ouagadougou.

MET (1994), « Plan d'Action National pour l'Environnement », OUAGA BURKINA.

MET Loi n002/94 A.D.P (du 19 Janvier 1994), « Code de l’Environnement », Ouagadougou.

Mostert W., Wiart A., Barrelon P., Doublier M., (1998), « Programme Régional de l'Utilisation du Butane dans les Pays du CILSS comme Combustible de Substitution au Charbon de bois et au Bois de chauffe », Tome 1, CILSS : Ouagadougou.

OUÉDRAOGO Boukary, (2002) « Éléments Économiques pour la Gestion de l'Offre et de la Demande du Bois-énergie dans la Région de Ouagadougou.», 380 pages, Thèse de doctorat Unique, Université de Ouagadougou-Université/Montesquieu Bordeaux IV. 
OUÉDRAOGO Boukary, (1996). « Analyse économique de l'approvisionnement de Ouagadougou en bois de feu. Une contribution a la gestion du patrimoine forestier.», 120 Pages, Mémoire de DEA/ PTCI, Université de Ouagadougou

OUÉDRAOGO Marie-Michèle, (1974). «L'Approvisionnement de Ouagadougou en Produits Vivriers, en Eau et en Bois ", Thèse de Doctorat de $3^{\circ}$ Cycle, CVRS : Université Bordeaux III, 353 pages.

PNUD/BKF/89/011, (1993), « Schéma directeur de l'aménagement des forêts naturelles autour de Ouagadougou. », BKF/89/011 : Ouagadougou.

Pearce David W., Turner Kerry R. (1990), Economics of natural resources and the environnement, Johns Hopkins University Press.

PNUD/BKF/93/003, (1994), « Aménagement des forêts naturelles pour la sauvegarde et la production du bois. », BKF: Ouagadougou.

PNUD/FAO/BKF/85/011 (BERMBA G. Francis et OUEDRAOGO S. Robert), (1992), « Prix de revient du bois et rentabilité économique du projet BKF/89/011», Ouagadougou: FAO.

PNUD/FAO/BKF/85/011 (TAIEB SELMI Mohamed), (1992), « Avant-Projet de Schéma Directeur d'Aménagement de Formations Forestières Naturelles dans un Univers de Six Millions d'Hectares Environ Autour de la Ville de Ouagadougou», Ouagadougou: FAO.

PNUD/FAO/BKF/85/011 (ZIDA Bertrand), (Juillet 1991), « Production, Commercialisation et consommation des combustibles ligneux, Région et ville de Ouagadougou 1990-2010 ", Ouagadougou.

PNUD/FAO/BKF/85/011, (1992), « Cartes des Etats de Surfaces au 1/200 000 pour 14 Provinces Autours de Ouagadougou :Méthodologie et Tableaux Bruts des Planimètres », FAO : Rome. PNUD/FAO/BKF/89/011(OUEDRAOGO Kimsé), (1995), « Plan de travail de 1995 », BKF: Ouagadougou. PNUD/FAO/BKF/89/011, (1993), « Rapport final de la mission d'évaluation tripartite», Québec (Canada).

RPTES (OUEDRAOGO Boukary, Juin 2000), « Demande Artisanale du bois-énergie comme Facteur de Production : cas des dolotières, restaurateurs, grilleurs de viande, fondeurs de bronze et d'aluminium», Ouagadougou.

RPTES/APEX (COMPAORE , 2000), « Etude sur l'approvisionnement des centres urbains du Burkina Faso en charbon de bois : Villes de Ouagadougou, Koudougou et Ouahigouya », Ouagadougou.

RPTES/CEEF (ILBOUDO Jean de Dieu, Août 2000), « Etude sur la filière commerciale du boisénergie au Burkina Faso », Ouagadougou.

RPTES/CEEF (ILBOUDO Jean de Dieu, Juin 1999), « Enquête de ravitaillement de Ouagadougou en combustible ligneux, réalisée du 18 au 24 Juin 1999.», Ouagadougou.

Sharma Narendra P., (1992), « Managing the world's forests: looking for balance between conservation and development», printed in the United States of America, the Kendall/Hunt Publishing Campant-(IBRD)/World Bank..

Sharma Narendra, RIETBERGEN S., HEIMO Claude R. et PATEL J., 1994. « A Strategy for the forest sector in Sub-saharian Africa», (IBRD)/World Bank washington, USA.

Some C., Tankoano M. J., Koulibaly R., et Thiombiano T., (1993) «Séminaire sur la Problématique de l'Approvisionnement de la Ville de Ouagadougou en Combustible Ligneux», Document de base, Ouagadougou. 
Sow Hamed, (1990), « Le bois-énergie au Sahel », ACCT-ATC- KARTHALA, Paris.

SSA/M.A.,(1998); « Rapport Général : Enquête Nationale de Statistiques Agricoles (ENSA) de $1993 » ;$ M.A., Ouagadougou.

Tubutin D. et Thiltges E., (1992), « Relations entre croissance démographique et environnement: du doctrinal à l'empirique », Revue Tiers Monde, XXXIII(130).

Vallin Jacques, (1994), «Mouvements démographiques et développement », Revue problèmes économiques, 2(379).

Yameogo K. Emmanuel (1995), « Impact de la dévaluation du FCFA et des évolutions récentes des prix sur les prévisions de recettes et de charges de gestion des chantiers en phases d'exploitation commerciales », mémoire de DEA, Ouagadougou: FASEG.

ZATU N AN VIII-039 BIS/FP/PRESS, (JUIN 1991), «Textes Portant Réorganisation Agraire et Foncière», OUAGADOUGOU.

\section{ANNEXES}

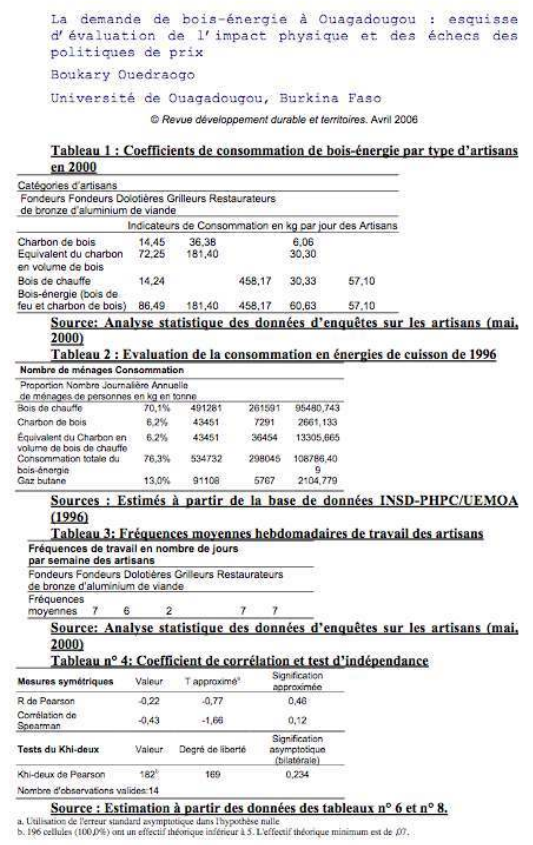

\section{NOTES}

1. Nous avons procédé à des visites auprès d'une vingtaine d'artisans toutes catégories confondues, dans le but de recenser leurs facteurs de production et de comprendre leur processus de production, avant d'établir les questionnaires et d'identifier leur localisation géographique dans la ville de Ouagadougou. Cela a eu l'avantage de favoriser une stratification conséquente des zones de sondage. 
2. Le coefficient de consommation des dolotières est donné par préparation de dolo et non par jour.

\section{RÉSUMÉS}

Cet article vise à évaluer non seulement la demande de bois-énergie de la ville de Ouagadougou, l'impact de cette demande sur le patrimoine forestier de l'aire d'approvisionnement de cette ville, mais aussi l'esquisse d'une évaluation des échecs des politiques de prix du bois-énergie. Les méthodes d'évaluation de la consommation de cette source d'énergie sont passées en revue au regard des conditions d'offre de la ressource et des données existantes sur la demande de cette ressource. Les données sur les inventaires forestiers de l'aire d'approvisionnement de Ouagadougou en bois-énergie, ainsi que des enquêtes sur les ménages et les artisans ont permis d'estimer la consommation de Ouagadougou en bois-énergie et son impact sur l'épuisement des ressources forestières du pays.

This paper aims at evaluating not only the wood-energy demand of Ouagadougou, the physic impact of this city wood energy consumption on the forest of its provisioning area, together with the causes of wood-energy price policy failures. The evaluation's methods of wood energy consumption are reviewed in comparison with the resource supply conditions and the availability on data of wood resource demand. The data on the forest inventories of the woodenergy provisioning surface of Ouagadougou, as well as investigations into the households and the craftsmen allows estimating the consumption of Ouagadougou in wood-energy and its impact on the forest resources' depletion. some complementary analysis have been done on the main causes of wood energy price policy failures.

\section{AUTEUR}

\section{BOUKARY OUEDRAOGO}

Université de Ouagadougou, Burkina Faso 